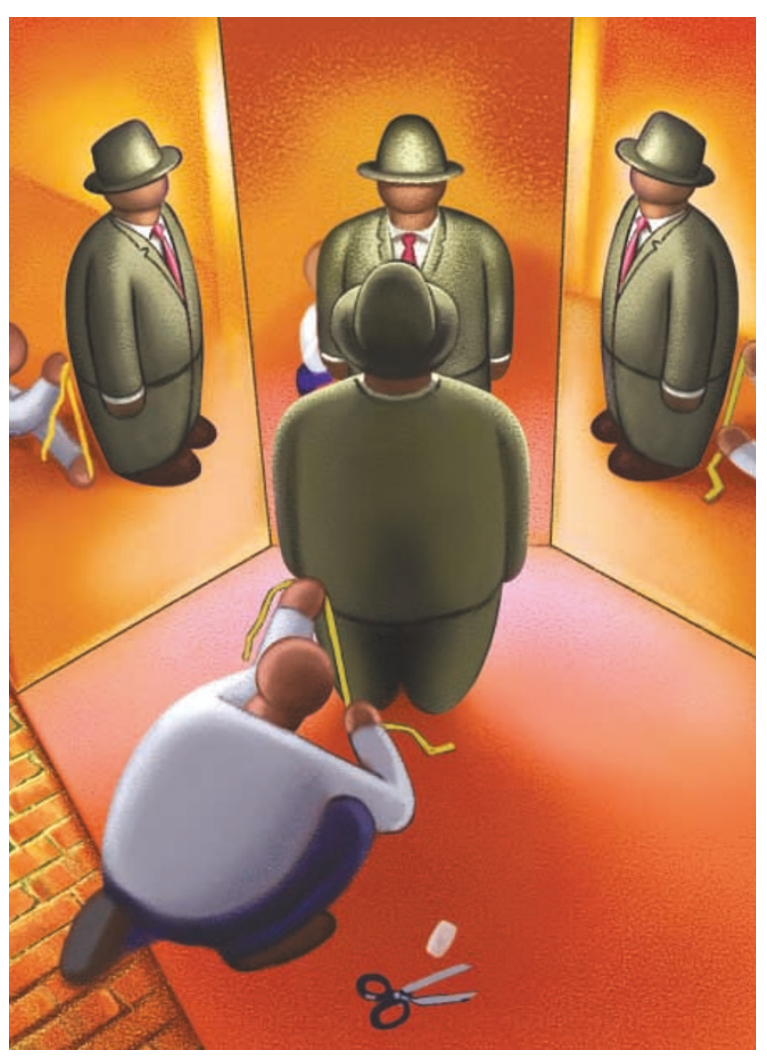

NEURODEGENERATIVE DISEASE

\section{Ataxin the brain}

Polyglutamine diseases - presently untreatable human neurodegenerative disorders that include Huntington's disease and several types of spinocerebellar ataxia (SCA) - are associated with expansion of CAG trinucleotide repeats. In SCA type 1, elevated polyglutamine concentration occurs in the kinase ataxin-1, and accumulation of this protein is often seen in the neuronal nuclei. Now, reports published in 8 May issue of Neuron and 16 May issue of Cell demonstrate that phosphorylation of ataxin-1 plays a crucial role in SCA type 1 pathogenesis.

In neurons of SCA1 transgenic mice, a mutated gene encoding ataxin-1 directs production of the protein with an increasing number of consecutive residues of the amino acid glutamine, which leads to the formation of nuclear inclusions. Although the glutamine repeats certainly contribute to protein misfolding and disease progression, recent research has indicated that key interactions between ataxin- 1 and other proteins could involve residues of ataxin-1 other than the polyglutamine tract.

\section{Bespoke binding}

Unravelling the features that give rise to specific and high-affinity binding of ligands by proteins is crucial for both advancing the basic understanding of molecular biological interactions, and for a number of applications in biotechnology and medicine. Reporting in the 9 May issue of Nature, Looger et al. describe a new computational approach for designing receptor and sensor proteins with specificity and affinity for desired small molecules.

Looger et al. started with a series of bacterial periplasmic binding proteins (PBPs) - Venusflytrap-like receptors comprising two domains that snap shut on binding their ligand. This ligand binding transmits a signal through the receptor that activates a pathway resulting in changes in bacterial gene expression. The researchers set out to redesign the binding site of these PBPs so that they would specifically bind a range of small molecules: the explosive trinitrotoluene (TNT), the sugar L-lactate and the hormone serotonin (5-HT). These ligands are structurally and chemically diverse, both from one another and the natural ligands of the PBPs.

The first step in the design process was to use an algorithm to identify the PBP binding site, and to place, in a computer model, a 'virtual' ligand in the binding site. Then, amino acids making up the binding site were sequentially mutated to find sequences that provided complementary surfaces to the desired ligand. This creates $10^{23}$ possible sequences, a number much greater than can be screened in vitro. In addition, the orientation of the ligand, and the conformations that the amino-acid side-chains could potentially take, were factored in, thereby creating further diversity. In order to search through these truly vast numbers of proteins, Looger $e t$ al. used an enhanced version of a search algorithm, which identified 17 virtually designed receptors that were selected and synthesized for experimental testing.

The designed PBPs were put through their paces in two experimental set-ups. In the first, the engineered PBPs were attached to a dye molecule that fluoresced on ligand binding by the PBP. By measuring levels of fluorescence, novel PBPs that bound TNT, L-lactate and 5-HT with affinities up
Teams led by Orr and Zoghbi assessed whether phosphorylation has a role in regulating the function and pathogenicity of ataxin-1. They report in Neuron that one specific amino acid of ataxin- 1 - serine 776 - is a site of phosphorylation. The removal of the phosphorylation site affected the cellular deposition of polyglutamineexpanded ataxin-1, which then failed to form nuclear inclusions in tissue culture cells. When this form of ataxin- 1 was expressed in mice, even though the ataxin-1 was found to be expressed within the neuronal nuclei, the mice exhibited a dramatic decrease in neuronal dysfunction. This indicates that phosphorylation of the specific serine residue on ataxin-1 plays a crucial role in modulating the ability of the mutant form of ataxin- 1 to induce neurodegeneration by influencing its normal interactions with other proteins and the formation of nuclear inclusions.

In the Cell paper, the researchers discovered that a multifunctional regulatory molecule, the 14-3-3 protein, mediates the neurotoxicity of ataxin- 1 by binding to and stabilizing the protein, thereby slowing its normal degradation. The further finding that the association of ataxin- 1 with $14-3-3$ is regulated by phosphorylation of another regulatory protein, $\mathrm{Akt}$, and that in a

Drosophila model of SCA1 both 14-3-3 and Akt modulate neurodegeneration, provides

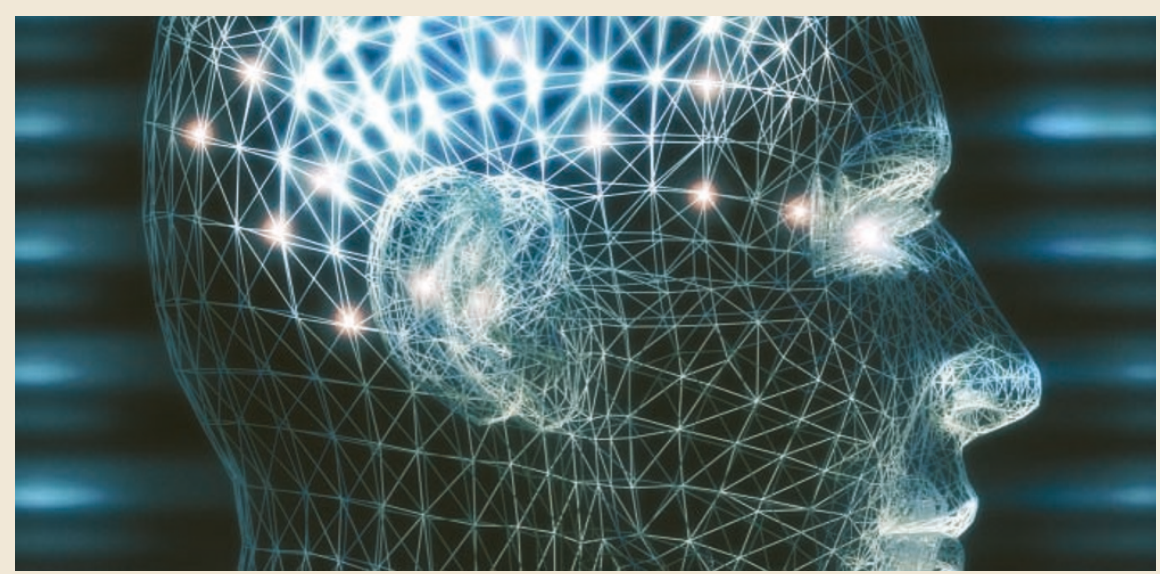


to those typical of antibody-ligand interactions were detected. In the other set of experiments, the selected PBPs were inserted into Escherichia coli such that the receptors were incorporated into a synthetic signalling pathway leading to the production of a reporter enzyme.

This approach is an important advance because of its generality, and the scope it provides for redesigning protein binding sites for ligands very dissimilar to the natural ligand of the protein. This was achieved in this study by taking into account both the orientation of the ligand and the myriad conformations that amino-acid side-chains within the binding site can adopt. Looking ahead, this method could have a wide range of applications, from improving chiral separations to developing molecular sensors for the presence of small-molecule biomarkers in clinical diagnostics, and perhaps ultimately in the design of catalytic proteins.

Daniel Jones

(2) References and links ORIGinAL ReSEARCh PAPER Looger, L. L., Dwyer, M. A., Smith, J. J. \& Hellinga, H. W. Computational design of receptor and sensor proteins with novel functions. Nature $\mathbf{4 2 3}$ 185-190 (2003)

FURTHER READING DeGrado, W. F. Biosensor design. Nature 423, 132-133 (2003)

further insight into SCA1 pathogenesis and identifies potential targets for therapeutic intervention.

This body of work shows that expansion of the polyglutamine tract or nuclear expression alone are not sufficient to cause disease, but that phosphorylation of ataxin-1 contributes to disease progression. The cellular proteins with which ataxin-1 normally interacts are likely to be important in the disease process, indicating that blocking the phosphorylation events could be a viable treatment. This is particularly exciting because kinases are tractable targets in drug discovery.

Melanie Brazil

(i) References and links ORIGINAL RESEARCH PAPERS Emamiam, E. S. et al. Serine 776 of ataxin- 1 is critical for polyglutamine-induced disease in SCA1 transgenic mice. Neuron 38, 375-387 (2003)|Chen, H. K. et al. Neuron 38, 375-387 (2003) Chen. H.-K et Interaction of Akt-phosphorylated ataxin-1 with 14-3-3 mediates neurodegeneration in spinocerebellar ataxia type 1. Cell 113, 457-468 (2003) FURTHER READING Gusella, J. F. \& MacDonald, M. E. Molecular genetics: unmasking polyglutamine triggers in neurodegenerative disease. Nature Rev. Neurosci. 1, 109-115 (2000) Orr. H. T. \& Zoghbi, H. Y. SCA1 molecular genetics: Orr, H. T. \& Zoghbi, H. Y. SCA1 molecular gen
a history of a 13-year collaboration against glutamines. Hum. Mol. Genet. 10, 2307-2311 (2001) | Orr, H. T. The ins and outs of a polyglutamine neurodegenerative disease: spinocerebellar ataxia type 1 (SCA1). Neurobiol. Dis. 7, 129-134 (2000)

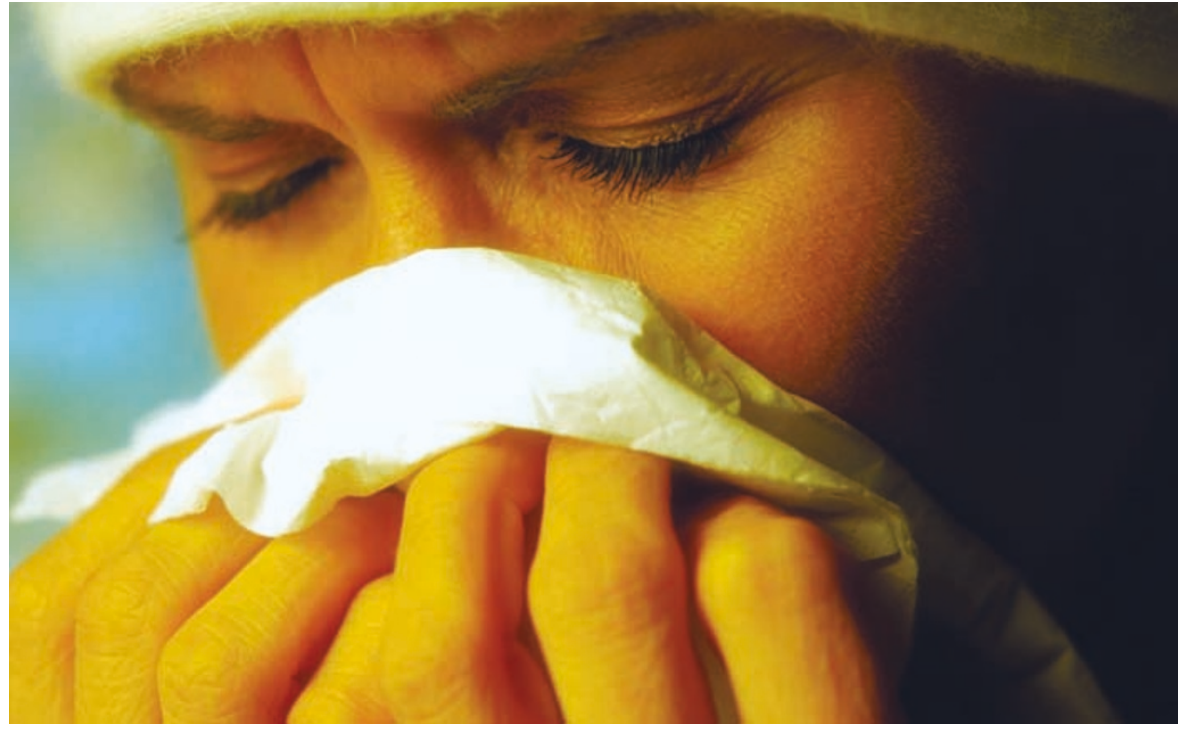

STRUCTURE-BASED DRUG DESIGN

\section{A common cure for SARS?}

The threat of a worldwide epidemic of severe acute respiratory syndrome (SARS) prompted an unprecedented global effort to identify its cause. Within weeks, a novel human coronavirus was identified as the root of the disease and its genome was sequenced, allowing researchers to identify crucial proteins of the SARS coronavirus (SARS-CoV) against which thousands of compounds are being screened to find new drugs. But a more directed approach to finding a cure has now been described in Science.

Rolf Hilgenfeld and colleagues focused their efforts on the viral main proteinase $\left(\mathrm{M}^{\mathrm{pro}}\right)$, which is necessary for the replication and transcription of coronaviruses, reasoning that it would be a good target for the development of drugs against SARS. Homology modelling was used to predict the structure of this crucial proteinase in SARS-CoV.

The researchers first determined the crystal structure of the main proteinase from a wellcharacterized coronavirus that causes cold symptoms in humans. This structure, together with the main proteinase structure from a related coronavirus that infects pigs, was used to construct a three-dimensional model of the SARS-CoV M ${ }^{\text {pro }}$. Computer modelling revealed that the structures of the substrate-binding regions of all three proteinases were highly conserved. Furthermore, the SARS-CoV M ${ }^{\text {pro }}$ was able to cleave a peptide corresponding to the autocatalytic site of the pig coronavirus proteinase in the test tube. Peptide inhibitors would therefore be expected to bind the different coronavirus main proteinases in the same manner.

But how could this information be used to find an effective drug for SARS? The researchers looked at a peptide inhibitor called AG7088, which targets a human rhinovirus proteinase that is distantly related to the coronavirus main proteinase, and is being clinically tested by
Pfizer for treating colds. They compared the structure of the rhinovirus enzyme bound to AG7088 with the structure of the pig coronavirus main proteinase bound to a substratemimic inhibitor. And the result? A surprising similarity in the way that the inhibitors slotted into their respective binding sites.

A key difference in the architecture of one subsite of the proteinase-binding sites led the researchers to suggest that AG7088 itself would be unlikely to switch off a coronavirus proteinase. Nonetheless, they proposed that it would provide a good starting point for designing drugs against SARS. Two days after this article was published, Pfizer announced that it had donated several compounds to the United States Army Medical Research Institute of Infectious Diseases (USAMRIID) and the National Institute of Allergy and Infectious Diseases (NIAID) to be tested for activity against SARS. According to Pfizer, a number of these compounds had shown moderate in vitro activity against SARS, including AG7088.

Although it remains to be shown whether AG7088 will have an effect in SARS patients, these results confirm the prediction of Hilgenfeld and colleagues that it might provide a valuable framework from which to develop drugs to target SARS. Furthermore, as AG7088 has already made it into clinical trials as a cold remedy, molecules developed from it are more likely to be both safe and efficacious.

Clare Ellis

(2) References and links

ORIGINAL RESEARCH PAPER Anand, K., Ziebuhr, P.

Wadhwani, P., Mesters, J. R. \& Hilgenfeld, R. Coronavirus main proteinase (3Cpro) structure: basis for design of anti-SARS drugs. Science 2003 May 13 (DOI: 10.1126/science.1085658) FURTHER READING Drosten, C. et al. Identification of a novel coronavirus in patients with severe acute respiratory syndrome. N. Engl J. Med 348, 1967-1976 (2003) | Ksiazek, T. G. et al. A nove syndrome. N. Engl. J. Med. 348, 1953-1966 (2003) 